\title{
Rare kaon decays and CP violation
}

\section{Antonio Pich*}

IFIC, Universitat de València - CSIC

Parc Científic, Catedrático José Beltrán 2, E-46980 Paterna, Spain

E-mail: Antonio.Pich@ific.uv.es

Owing to the strong suppression of flavour-changing neutral-current transitions in the Standard Model, rare kaon decays constitute a superb tool to constrain hypothetical new-physics interactions. At the same time, they provide many interesting tests of the Standard Model itself, being sensitive both to short-distance electroweak scales and to the long-distance QCD dynamics. A brief overview of the current status is presented. The Standard Model prediction for the direct $\mathrm{CP}$-violating ratio $\varepsilon^{\prime} / \varepsilon$ is also discussed.

18th International Conference on B-Physics at Frontier Machines - Beauty2019-

29 September $/ 4$ October, 2019

Ljubljana, Slovenia

${ }^{*}$ Speaker. 


\section{Introduction}

The investigation of kaon decays has uncovered many fundamental ingredients of the electroweak theory, such as flavour quantum numbers, meson-antimeson mixing, parity violation, $\mathrm{CP}$ violation, quark mixing, and the GIM mechanism [1]. The kaon decay amplitudes provide very interesting tests of the Standard Model (SM), since they involve an intricate interplay between weak, electromagnetic and strong interactions. Moreover, rare kaon decays are sensitive to short-distance scales $\left(c, t, W^{ \pm}, Z\right)$ and have the potential to unravel new physics (NP) beyond the SM. In particular, high-precision searches for lepton-flavour violation (LFV) beyond the $10^{-10}$ level $\left[\operatorname{Br}\left(K_{L} \rightarrow e^{ \pm} \mu^{\mp}\right)<4.7 \cdot 10^{-12}\right.$ [2], $\operatorname{Br}\left(K_{L} \rightarrow e^{ \pm} e^{ \pm} \mu^{\mp} \mu^{\mp}\right)<4.12 \cdot 10^{-11}$ [3], $\operatorname{Br}\left(K^{+} \rightarrow \pi^{+} \mu^{+} e^{-}\right)<1.3 \cdot 10^{-11}$ [4], $\operatorname{Br}\left(K^{+} \rightarrow \pi^{+} \mu^{-} e^{+}\right)<5.2 \cdot 10^{-10}$ [5] (90\% CL)] are actually exploring energy scales above the $10 \mathrm{TeV}$ region. In addition, the mechanism of $\mathrm{CP}$ violation can be accurately tested, both in the observed kaon decay modes and through still undetected processes such as $K_{L} \rightarrow \pi^{0} v \bar{v}$.

The fundamental flavour-changing transitions among the constituent quarks are characterized by the electroweak scale, but the corresponding hadronic amplitudes are governed by the longdistance behaviour of the strong interactions, i.e., the confinement regime of QCD. In kaon decays, the presence of widely separated mass scales $\left(m_{\pi}<m_{K} \ll M_{W}\right)$ amplifies the QCD corrections with logarithms of the large mass ratios. Using the operator product expansion (OPE) and the renormalization group to integrate out the heavy fields all the way down from $M_{W}$ to scales $\mu<m_{c}$, one gets an effective Lagrangian, defined in the three-flavour theory [6],

$$
\mathscr{L}_{\mathrm{eff}}^{\Delta S=1}=-\frac{G_{F}}{\sqrt{2}} V_{u d} V_{u s}^{*} \sum_{i} C_{i}(\mu) Q_{i}, \quad C_{i}(\mu)=z_{i}(\mu)-y_{i}(\mu) \frac{V_{t d} V_{t s}^{*}}{V_{u d} V_{u s}^{*}},
$$

which contains local four-fermion operators $Q_{i}$, constructed with the light degrees of freedom $(u, d$, $\left.s ; e, \mu, v_{\ell}\right)$, modulated by Wilson coefficients $C_{i}(\mu)$ that are functions of the heavy $(Z, W, t, b, c, \tau)$ masses and encode the short-distance logarithmic corrections. The violations of the CP symmetry originate in the $y_{i}(\mu)$ components, which are proportional to the top-quark mixing factors.

The coefficients $C_{i}(\mu)$ are currently known at the next-to-leading order (NLO) [7, 8, 9, 10], which includes all corrections of $\mathscr{O}\left(\alpha_{s}^{n} t^{n}\right)$ and $\mathscr{O}\left(\alpha_{s}^{n+1} t^{n}\right)$, with $t \equiv \log \left(M_{1} / M_{2}\right)$ the logarithm of any ratio of heavy mass scales $\left(M_{1,2} \geq \mu\right)$. The long-distance contributions from scales below the renormalization scale $\mu$ are contained in the non-perturbative matrix elements of the operators $Q_{i}$ between the initial and final hadronic states. These contributions should cancel exactly the renormalization scale (and scheme) dependence of the Wilson coefficients. Unfortunately, a rigorous analytic evaluation of the hadronic matrix elements, keeping full control of the QCD renormalization conventions, remains still a challenging task.

At low energies, one can use symmetry considerations to define another effective field theory in terms of the QCD Goldstone bosons $(\pi, K, \eta)$. Chiral Perturbation Theory $(\chi \mathrm{PT})[11,12]$ describes the dynamics of the pseudoscalar octet through a perturbative expansion in powers of momenta and quark masses over the chiral symmetry-breaking scale $\Lambda_{\chi} \sim 1 \mathrm{GeV}$. Chiral symmetry determines the allowed operators, while all short-distance information is encoded in their low-energy couplings (LECs) [13, 14]. At LO the most general effective Lagrangian, with the same $S U(3)_{L} \otimes S U(3)_{R}$ 


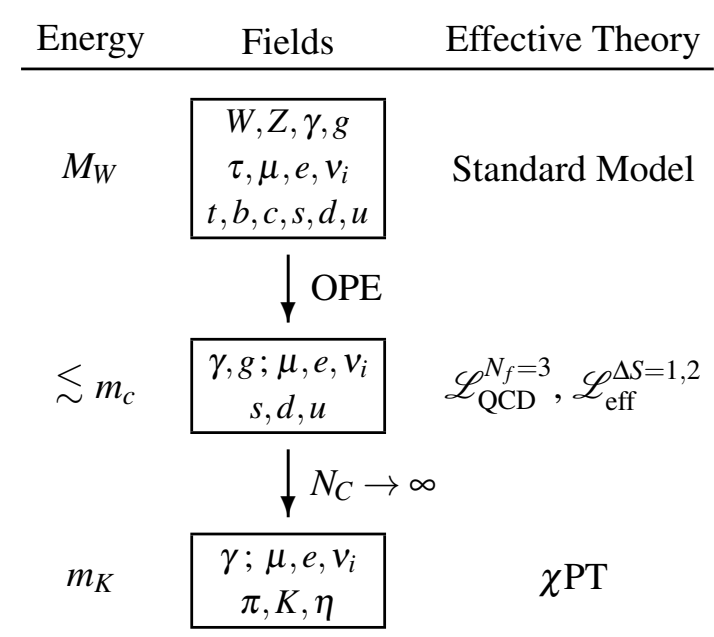

Figure 1: Evolution from $M_{W}$ to $m_{K}$.

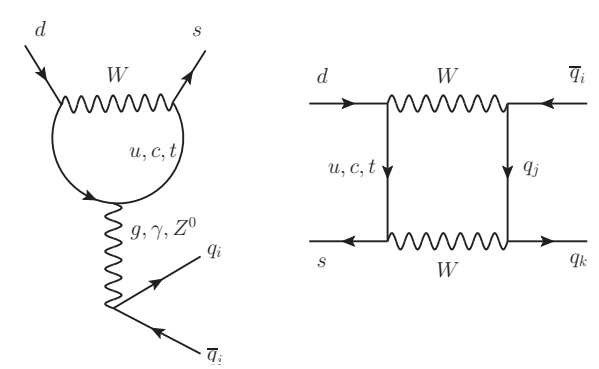

Figure 2: Short-distance diagrams.

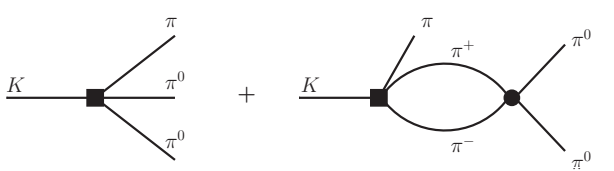

Figure 3: Long-distance diagrams.

transformation properties as the short-distance Lagrangian (1.1), contains three terms [1]:

$$
\mathscr{L}_{2}^{\Delta S=1}=-\frac{G_{F}}{\sqrt{2}} V_{u d} V_{u s}^{*}\left\{g_{8}\left\langle\lambda L_{\mu} L^{\mu}\right\rangle+g_{27}\left(L_{\mu 23} L_{11}^{\mu}+\frac{2}{3} L_{\mu 21} L_{13}^{\mu}\right)+e^{2} g_{8} g_{\mathrm{ew}} F^{6}\left\langle\lambda U^{\dagger} Q U\right\rangle\right\},
$$

where the $S U(3)$ matrix field $U \equiv \exp (i \vec{\lambda} \vec{\phi} / F)$ parametrizes the octet of Goldstone bosons, $L_{\mu}=$ $i F^{2} U^{\dagger} D_{\mu} U$ represents the $V-A$ currents, $\lambda \equiv\left(\lambda_{6}-i \lambda_{7}\right) / 2$ projects onto the $\bar{s} \rightarrow \bar{d}$ transition, $Q=\frac{1}{3} \operatorname{diag}(2,-1,-1)$ is the quark charge matrix and \langle\rangle denotes a 3 -dimensional flavour trace. The $\mathscr{O}\left(p^{2}\right)$ LECs $g_{8}$ and $g_{27}$ measure the strength of the two parts of $\mathscr{L}_{\text {eff }}^{\Delta S=1}$ transforming as $\left(8_{L}, 1_{R}\right)$ and $\left(27_{L}, 1_{R}\right)$, respectively, under chiral rotations, while the $g_{8} g_{\text {ew }}$ term is of $\mathscr{O}\left(e^{2} p^{0}\right)$ and accounts for the $\left(8_{L}, 8_{R}\right)$ piece induced by the electromagnetic penguin operators.

The $\chi \mathrm{PT}$ framework determines the most general form of the $K$ decay amplitudes, compatible with chiral symmetry, in terms of the LECs multiplying the relevant chiral operators. A firstprinciple calculation of these LECs would require to perform a non-perturbative matching between $\chi \mathrm{PT}$ and the underlying SM. While some lattice information is already available in the strong sector, the LECs can be determined phenomenologically and/or calculated in the limit of a large number of QCD colours $N_{C}$. Fig. 1 shows schematically the procedure used to evolve down from the electroweak scale, where the underlying flavour-changing processes take place (Fig. 2), to $m_{K}$. The short-distance logarithmic corrections $\log (M / \mu)$ are summed up with the OPE and the resulting effective Lagrangian $\mathscr{L}_{\text {eff }}^{\Delta S=1}$ is then matched into the low-energy $\chi$ PT formalism.

At NLO in the $\chi \mathrm{PT}$ expansion, one must consider tree-level contributions from additional operators of $\mathscr{O}\left(p^{4}\right)$ and $\mathscr{O}\left(e^{2} p^{2}\right)$, with their corresponding LECs, and quantum loops with the LO Lagrangian (1.2). These chiral loops (Fig. 3) generate non-polynomial contributions, with logarithms and threshold factors as required by unitarity. The loop corrections contain large infrared logarithms, $\log \left(\mu / m_{\pi}\right)$, and Goldstone re-scattering contributions (final-state interactions) that play a very important role in the kaon decay dynamics. 


\section{The CP-violating ratio $\varepsilon^{\prime} / \varepsilon$}

A tiny difference between the CP-violating ratios $\eta_{n m} \equiv \mathscr{M}\left[K_{L}^{0} \rightarrow \pi^{n} \pi^{m}\right] / \mathscr{M}\left[K_{S}^{0} \rightarrow \pi^{n} \pi^{m}\right] \approx$ $\varepsilon \approx 2.2 \times 10^{-3} \mathrm{e}^{i \pi / 4}$, where $n m=+-, 00$ denote the final pion charges, was first measured by the CERN NA31 experiment [15] and later confirmed at the 7.2 $\sigma$ level with the full data samples of NA31, NA48 and the Fermilab experiments E731 and KTeV [16]:

$$
\operatorname{Re}\left(\varepsilon^{\prime} / \varepsilon\right)=\frac{1}{3}\left(1-\left|\frac{\eta_{00}}{\eta_{+-}}\right|^{2}\right)=(16.6 \pm 2.3) \times 10^{-4} .
$$

This important measurement established the presence of direct $\mathrm{CP}$ violation in the decay amplitudes, confirming that $\mathrm{CP}$ violation is associated with a $\Delta S=1$ transition as predicted by the SM.

The first NLO theoretical predictions gave values of $\varepsilon^{\prime} / \varepsilon$ one order of magnitude smaller than (2.1), but it was soon realised that they were missing the important role of the final pion dynamics $[17,18,19]$. Once long-distance contributions are properly taken into account, the theoretical SM prediction turns out to be in good agreement with the experimental value, although the uncertainties are unfortunately large [20,21]:

$$
\operatorname{Re}\left(\varepsilon^{\prime} / \varepsilon\right)_{\mathrm{SM}}=(14 \pm 5) \times 10^{-4} .
$$

The underlying physics can be easily understood from the kaon data themselves. Owing to Bose symmetry, the two pions in the final state must be in a $I=0$ or $I=2$ configuration. In the absence of QCD corrections, the corresponding $K \rightarrow \pi \pi$ decay amplitudes $\mathscr{A}_{I} \equiv A_{I} \mathrm{e}^{i \delta_{I}}$ are predicted to differ only by a $\sqrt{2}$ factor. However, their measured ratio is 16 times larger than that (a truly spectacular enhancement generated by the strong forces):

$$
\omega \equiv \operatorname{Re}\left(A_{2}\right) / \operatorname{Re}\left(A_{0}\right) \approx 1 / 22, \quad \delta_{0}-\delta_{2} \approx 45^{\circ} .
$$

Moreover, they exhibit a huge phase-shift difference that manifests the relevance of final-state interactions and, therefore, the presence of large absorptive contributions to the $K \rightarrow \pi \pi$ amplitudes, specially to the isoscalar one. Writing $\mathscr{A}_{I}=\operatorname{Dis}\left(\mathscr{A}_{I}\right)+i \operatorname{Abs}\left(\mathscr{A}_{I}\right)$ and neglecting the small CP-odd components, the measured $\pi \pi$ scattering phase shifts at $\sqrt{s}=m_{K}$ imply that

$$
\operatorname{Abs}\left(\mathscr{A}_{0}\right) / \operatorname{Dis}\left(\mathscr{A}_{0}\right) \approx 0.82, \quad \operatorname{Abs}\left(\mathscr{A}_{2}\right) / \operatorname{Dis}\left(\mathscr{A}_{2}\right) \approx-0.15
$$

The direct CP-violating effect involves the interference between the two isospin amplitudes,

$$
\operatorname{Re}\left(\varepsilon^{\prime} / \varepsilon\right)=-\frac{\omega}{\sqrt{2}|\varepsilon|}\left[\frac{\operatorname{Im} A_{0}}{\operatorname{Re} A_{0}}-\frac{\operatorname{Im} A_{2}}{\operatorname{Re} A_{2}}\right]=-\frac{\omega_{+}}{\sqrt{2}|\varepsilon|}\left[\frac{\operatorname{Im} A_{0}^{(0)}}{\operatorname{Re} A_{0}^{(0)}}\left(1-\Omega_{\mathrm{eff}}\right)-\frac{\operatorname{Im} A_{2}^{\mathrm{emp}}}{\operatorname{Re} A_{2}^{(0)}}\right] .
$$

It is suppressed by the small ratio $\omega$ and, moreover, it is very sensitive to isospin-breaking (IB) corrections [22, 23, 24], parametrized by $\Omega_{\mathrm{eff}}=0.11 \pm 0.09$ [21], because small IB corrections to $A_{0}$ feed into the small amplitude $A_{2}$ enhanced by the large factor $1 / \omega$. In the right-hand side of Eq. (2.5), the (0) superscript indicates the isospin limit, $\omega_{+}=\operatorname{Re}\left(A_{2}^{+}\right) / \operatorname{Re}\left(A_{0}\right)$ is directly extracted from $K^{+} \rightarrow \pi^{+} \pi^{0}$ and $A_{2}^{\text {emp }}$ contains the electromagnetic-penguin contribution to $A_{2}$ (the remaining contributions are included in $\left.\Omega_{\mathrm{eff}}\right)$. 
Claims of too small SM values for $\varepsilon^{\prime} / \varepsilon$ usually originate from perturbative calculations that are unable to generate the physical phase shifts, i.e., they predict $\delta_{I}=0$ and, therefore, $\operatorname{Abs}\left(\mathscr{A}_{I}\right)=0$, failing completely to understand the empirical ratios (2.4). This unitarity pitfall implies also incorrect predictions for the dispersive components, since they are related by analyticity with the absorptive parts: a large absorptive contribution generates a large dispersive correction that is obviously missed in those calculations. This perturbative problem is more severe in $\varepsilon^{\prime} / \varepsilon$ because Eq. (2.5) involves a delicate numerical balance among the three contributing terms, and naive predictions sit precisely on a nearly-exact cancellation (a $40 \%$ positive correction to the first term enhances the whole result by one order of magnitude).

The $\varepsilon^{\prime} / \varepsilon$ anomaly was recently resurrected by the lattice RBC-UKQCD collaboration that reported $\operatorname{Re}\left(\varepsilon^{\prime} / \varepsilon\right)=(1.38 \pm 5.15 \pm 4.59) \times 10^{-4}[25,26]$. The uncertainties are still large, but the quite low central value implies a $2.1 \sigma$ deviation from the experimental measurement. This has triggered a revival of the old naive estimates [27, 28], some of them making also use of the lattice data [29, 30], and a large amount of NP explanations (a list of references is given in Refs. [20, 21]). However, it is premature to derive physics implications from the current lattice simulations, since they are still unable to reproduce the known phase shifts. While the lattice determination of $\delta_{2}$ is only $1 \sigma$ away from its physical value, $\delta_{0}$ disagrees with the experimental result by $2.9 \sigma$, a much larger discrepancy that the one quoted for $\varepsilon^{\prime} / \varepsilon$. Obviously, nobody suggests a NP contribution to the $\pi \pi$ elastic scattering phase shifts. The RBC-UKQCD collaboration is actively working in order to improve the present situation.

\section{Rare kaon decays in the SM}

Kaon decays mediated by flavour-changing neutral currents (FCNCs) are strongly suppressed in the SM and, therefore, are very sensitive to NP effects. In the SM, most of these processes are dominated by long-distance contributions, making quite challenging their precise theoretical understanding. However, there are also decays governed by short-distance amplitudes, such as $K \rightarrow \pi v \bar{v}$.

\section{1 $\mathrm{K}^{0} \rightarrow \gamma \gamma$ and $\mathrm{K}^{0} \rightarrow \ell^{+} \ell^{-}$}

At $\mathscr{O}\left(p^{4}\right)$ in the $\chi \mathrm{PT}$ expansion, the symmetry constraints do not allow any local $K_{1}^{0} \gamma \gamma$ vertex $\left(K_{1,2}^{0}\right.$ denote the CP-even and CP-odd neutral kaon states). The decay $K_{S}^{0} \rightarrow \gamma \gamma$ proceeds then through a one-loop amplitude, with virtual $\pi^{+} \pi^{-}$or $K^{+} K^{-}$pairs (Fig. 4), which is necessarily finite because there are no counterterms to renormalize divergences. The resulting prediction, $\operatorname{Br}\left(K_{S}^{0} \rightarrow \gamma \gamma\right)=2.0 \times 10^{-6}[31,32]$, is slightly lower than the experimental value $\operatorname{Br}\left(K_{S}^{0} \rightarrow \gamma \gamma\right)=$ $(2.63 \pm 0.17) \times 10^{-6}[16]$. Full agreement is obtained at $\mathscr{O}\left(p^{6}\right)$, once rescattering corrections $\left(K_{S}^{0} \rightarrow \pi \pi \rightarrow \pi^{+} \pi^{-} \rightarrow \gamma \gamma\right)$ are included [33].

The 2-loop amplitude $K_{S}^{0} \rightarrow \gamma^{*} \gamma^{*} \rightarrow \ell^{+} \ell^{-}$(Fig. 5) is also finite [34] because chiral symmetry forbids any $\mathrm{CP}$-invariant local contribution at this order. The predicted rates, $\operatorname{Br}\left(K_{S}^{0} \rightarrow e^{+} e^{-}\right)=$ $2.1 \times 10^{-14}$ and $\operatorname{Br}\left(K_{S}^{0} \rightarrow \mu^{+} \mu^{-}\right)=5.1 \times 10^{-12}$ [34], are well below the experimental upper bounds $\operatorname{Br}\left(K_{S}^{0} \rightarrow e^{+} e^{-}\right)<9 \times 10^{-9}$ [35] and $\operatorname{Br}\left(K_{S}^{0} \rightarrow \mu^{+} \mu^{-}\right)<2.1 \times 10^{-10}$ [36, 37] (90\% CL). This calculation allows us to compute the longitudinal polarization $P_{L}$ of either muon in the decay 


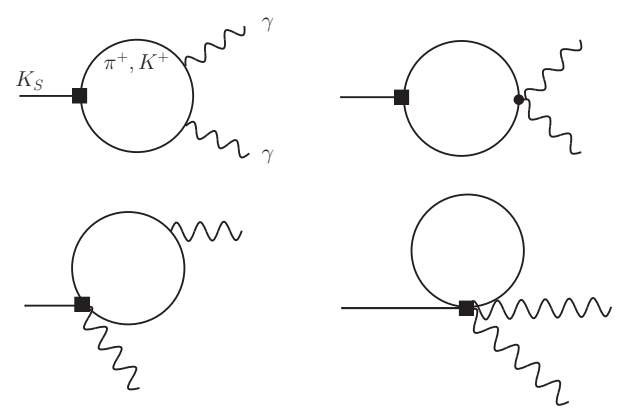

Figure 4: Lowest-order contributions to $K_{S} \rightarrow \gamma \gamma$.

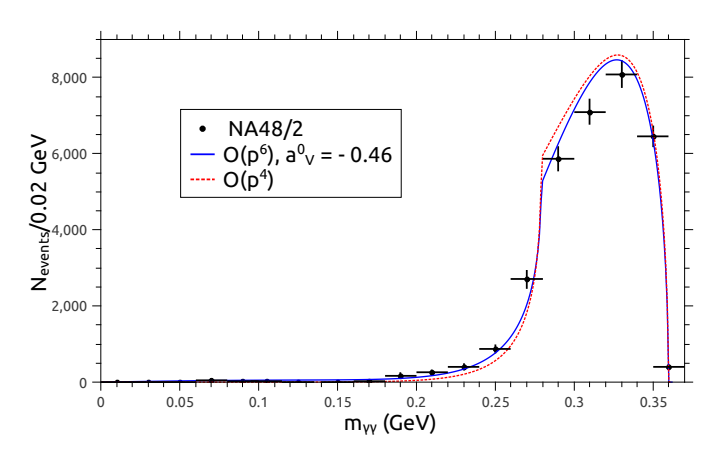

Figure 6: $K_{L} \rightarrow \pi^{0} \gamma \gamma$ spectra at $\mathscr{O}\left(p^{4}\right)$ and $\mathscr{O}\left(p^{6}\right)$ in $\chi$ PT. The data are from Ref. [41].

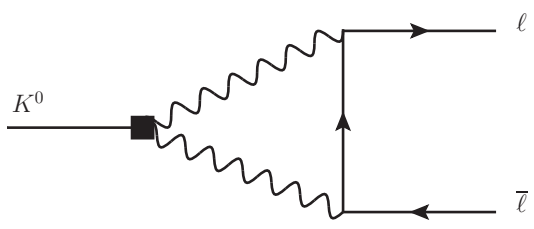

Figure 5: $2 \gamma$ contribution to $K^{0} \rightarrow \ell^{+} \ell^{-}$.

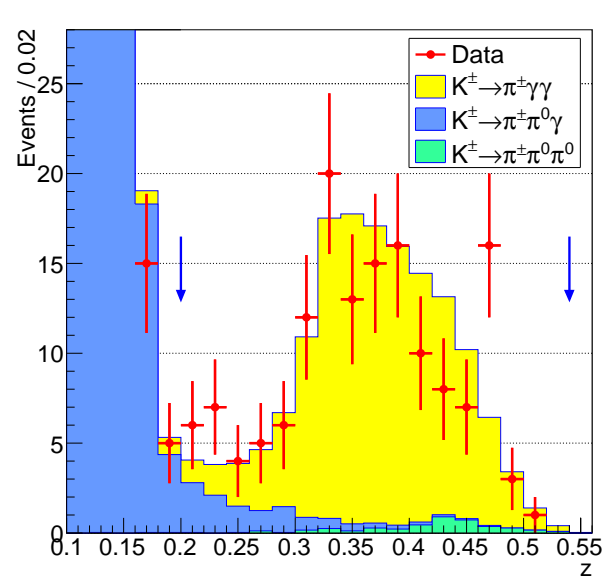

Figure 7: Measured $K^{ \pm} \rightarrow \pi^{ \pm} \gamma \gamma$ spectrum and estimated signal from an $\mathscr{O}\left(p^{6}\right) \chi \mathrm{PT}$ fit [47].

$K_{L}^{0} \rightarrow \mu^{+} \mu^{-}$, a CP-violating observable which in the SM is dominated by indirect CP violation from $K^{0}-\bar{K}^{0}$ mixing. One finds $\left|P_{L}\right|=(2.6 \pm 0.4) \times 10^{-3}$ [34].

\section{$3.2 \mathrm{~K} \rightarrow \pi \gamma \gamma$}

Again, the symmetry constraints do not allow any tree-level contribution to $K_{2}^{0} \rightarrow \pi^{0} \gamma \gamma$ from $\mathscr{O}\left(p^{4}\right)$ terms in the $\chi \mathrm{PT}$ Lagrangian. The decay amplitude is therefore determined by a finite loop calculation $[38,39,40]$. Due to the large absorptive $\pi^{+} \pi^{-}$contribution, the spectrum in the invariant mass of the two photons is predicted to have a very characteristic behaviour (dotted line in Fig. 6), peaked at high values of $m_{\gamma \gamma}$. The agreement with the measured distribution [41] is remarkably good. However, the $\mathscr{O}\left(p^{4}\right)$ prediction for the rate, $\operatorname{Br}\left(K_{L} \rightarrow \pi^{0} \gamma \gamma\right)=6.8 \times 10^{-7}$ [38], is significantly smaller than the present PDG average, $\operatorname{Br}\left(K_{L}^{0} \rightarrow \pi^{0} \gamma \gamma\right)=(1.27 \pm 0.03) \times 10^{-6}[16]$, indicating that higher-order corrections are sizeable. Unitarity corrections from $K_{L}^{0} \rightarrow \pi^{+} \pi^{-} \pi^{0}$ $[42,43]$ and local vector-exchange contributions $[42,44]$ restore the agreement at $\mathscr{O}\left(p^{6}\right)$.

A quite similar spectrum is predicted [45] for the charged mode $K^{ \pm} \rightarrow \pi^{ \pm} \gamma \gamma$, but in this case there is a free LEC already at $\mathscr{O}\left(p^{4}\right)$. Corrections of $\mathscr{O}\left(p^{6}\right)$ have been also investigated [46]. Both the measured spectrum and the rate can be correctly reproduced [47], as illustrated in Fig. 7. 


\section{3 $\mathrm{K}_{\mathrm{L}}^{0} \rightarrow \pi^{0} \mathrm{e}^{+} \mathrm{e}^{-}$}

This decay is an interesting process in looking for new CP-violating signatures, because $K_{2}^{0} \rightarrow$ $\pi^{0} \gamma^{*}$ violates CP $[45,48]$. The CP-conserving amplitude proceeds through a $2 \gamma$ intermediate state and is suppressed by an additional power of $\alpha$. Using the $K_{L}^{0} \rightarrow \pi^{0} \gamma \gamma$ data, the CP-conserving rate is found to be below $10^{-12}$ [1]. The $K_{L}^{0} \rightarrow \pi^{0} e^{+} e^{-}$transition is then dominated by the $\mathscr{O}(\alpha) \mathrm{CP}-$ violating contributions [45], both from $K^{0}-\bar{K}^{0}$ mixing and direct $\mathrm{CP}$ violation. The estimated rate, $\operatorname{Br}\left(K_{L} \rightarrow \pi^{0} e^{+} e^{-}\right)=(3.1 \pm 0.9) \times 10^{-11}[1,49,50]$, is only a factor 10 smaller than the present (90\% CL) upper bound of $2.8 \times 10^{-10}$ [51] and should be reachable in the near future.

\section{$3.4 \mathrm{~K} \rightarrow \pi v \bar{v}$}

Long-distance effects play a negligible role in $K^{+} \rightarrow \pi^{+} v \bar{v}$ and $K_{L}^{0} \rightarrow \pi^{0} v \bar{v}$. These processes are dominated by short-distance loops ( $Z$ penguin, $W$ box), involving the heavy top quark. The $K^{+}$ decay mode receives also sizeable contributions from internal charm-quark exchanges. The decay amplitudes are proportional to the hadronic matrix element of the $\Delta S=1$ vector current, which (assuming isospin symmetry) can be obtained from $K_{\ell 3}$ decays:

$$
T(K \rightarrow \pi \nu \bar{v}) \sim \sum_{i=c, t} F\left(V_{i d} V_{i s}^{*} ; x_{i}\right)\left(\bar{v}_{L} \gamma_{\mu} v_{L}\right)\left\langle\pi\left|\bar{s}_{L} \gamma^{\mu} d_{L}\right| K\right\rangle, \quad x_{i} \equiv m_{i}^{2} / M_{W}^{2} .
$$

The small long-distance and isospin-violating corrections can be estimated within $\chi \mathrm{PT}$. The $K_{L}^{0} \rightarrow$ $\pi^{0} v \bar{v}$ transition violates $\mathrm{CP}$ and is completely dominated by direct $\mathrm{CP}$ violation, the contribution from $K^{0}-\bar{K}^{0}$ mixing being only of the order of $1 \%$. Taking the CKM inputs from global fits, one predicts $\operatorname{Br}\left(K_{L}^{0} \rightarrow \pi^{0} v \bar{v}\right)=(2.9 \pm 0.3) \times 10^{-11}$ and $\operatorname{Br}\left(K^{+} \rightarrow \pi^{+} v \bar{v}\right)=(8.5 \pm 0.6) \times 10^{-11}$ $[52,53,54]$. The uncertainties are largely parametrical, due to CKM input, $m_{c}, m_{t}$ and $\alpha_{s}\left(M_{Z}\right)$.

The current (90\% CL) upper bounds on the charged [55] and neutral [56] modes are

$$
\operatorname{Br}\left(K^{+} \rightarrow \pi^{+} v \bar{v}\right)<1.85 \times 10^{-10}, \quad \operatorname{Br}\left(K_{L}^{0} \rightarrow \pi^{0} v \bar{v}\right)<3.0 \times 10^{-9} .
$$

The ongoing CERN NA62 experiment aims to reach $\mathscr{O}(100) K^{+} \rightarrow \pi^{+} v \bar{v}$ events (assuming SM rates), while increased sensitivities on the $K_{L}^{0} \rightarrow \pi^{0} v \bar{v}$ mode are expected to be achieved by the KOTO experiment at J-PARC.

\section{Constraints on scalar leptoquarks from rare kaon decays}

Rare kaon decays put strong constraints on NP interactions with non-trivial flavour dynamics. As an illustration, and motivated by the flavour anomalies reported recently in $B$ decays [57], let us consider the implications of kaon data on generic couplings of hypothetical scalar leptoquarks (LQs) to the SM fermions [58]:

$$
\mathscr{L}_{\mathrm{LQ}}=\overline{Q^{c}} i \tau_{2} y_{S_{1}} L S_{1}+\overline{d_{R}^{c}} y_{\tilde{S}_{1}} \ell_{R} \tilde{S}_{1}+\bar{\ell}_{R} y_{R_{2}} R_{2}^{\dagger} Q-\bar{d}_{R} y_{\tilde{R}_{2}} \tilde{R}_{2}^{T} i \tau_{2} L+\overline{Q^{c}} y_{S_{3}} i \tau_{2} \tau \cdot \mathbf{S}_{3} L+\text { h.c. }
$$

We have included the five possible types of scalar LQs, coupling to SM particles, with the following $S U(3)_{C} \otimes S U(2)_{L} \otimes U(1)_{Y}$ quantum numbers [59, 60]: $S_{1}(\overline{\mathbf{3}}, \mathbf{1}, 1 / 3), \tilde{S}_{1}(\overline{\mathbf{3}}, \mathbf{1}, 4 / 3), R_{2}(\mathbf{3}, \mathbf{2}, 7 / 6)$, $\tilde{R}_{2}(\mathbf{3}, 2,1 / 6)$ and $S_{3}(\overline{\mathbf{3}}, \mathbf{3}, 1 / 3) . Q$ and $L$ are the left-handed quark and lepton doublets, $d_{R}$ and $\ell_{R}$ the corresponding right-handed singlets and $f^{c} \equiv \mathscr{C} \bar{f}^{T}$ indicates the charge-conjugated field of the 

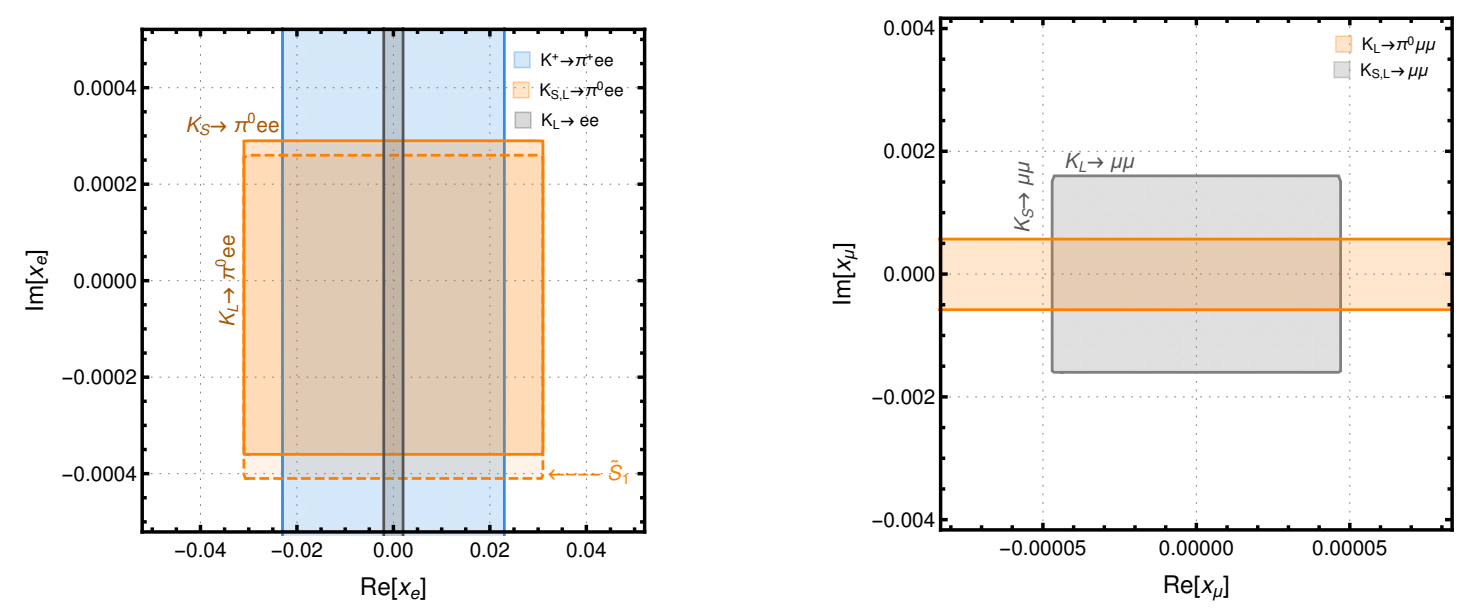

Figure 8: Allowed regions in the plane $\left(\operatorname{Re}\left[x_{\ell}\right], \operatorname{Im}\left[x_{\ell}\right]\right)$, arising from leptonic and rare semileptonic kaon decays, for the electron (left panel) and muon (right panel) channels [58].

fermion $f$. All fermion fields carry flavour indices and $y_{L Q}$ are arbitrary Yukawa matrices in flavour space. Eq. (4.1) only displays those couplings relevant for kaon decays.

The exchange of a heavy LQ between two fermionic currents induces tree-level contributions to the FCNC transitions $K^{0} \rightarrow \ell^{+} \ell^{-}$and $K \rightarrow \pi \ell^{+} \ell^{-}$. They are governed by the following combinations of LQ parameters:

$$
x_{e}=\left(\frac{1 \mathrm{TeV}}{M_{\mathrm{LQ}}}\right)^{2} \times\left\{\begin{array}{l}
y_{\mathrm{LQ}}^{11}\left(y_{\mathrm{LQ}}^{12}\right)^{*} \\
y_{\mathrm{LQ}}^{11}\left(y_{\mathrm{LQ}}^{21}\right)^{*}
\end{array}, \quad x_{\mu}=\left(\frac{1 \mathrm{TeV}}{M_{\mathrm{LQ}}}\right)^{2} \times\left\{\begin{array}{l}
y_{\mathrm{LQ}}^{21}\left(y_{\mathrm{LQ}}^{22}\right)^{*} \\
y_{\mathrm{LQ}}^{12}\left(y_{\mathrm{LQ}}^{22}\right)^{*}
\end{array}\right.\right.
$$

where $x_{e}$ and $x_{\mu}$ correspond to the electron and muon modes, respectively. The first line in the brackets corresponds to $R_{2}$, while the second line refers to $\tilde{R}_{2}, \tilde{S}_{1}$ and $(4 \times) S_{3}$ (the LQ $S_{1}$ does not contribute at tree level to these processes). The current constraints on $x_{\ell}$ from different kaon decay modes are displayed in Fig. 8 for the electron (left panel) and muon (right panel) final states. The $K_{S}^{0}$ and $K_{L}^{0}$ decays are complementary, providing separate access to both the real and imaginary parts of the NP couplings, while the decays of the charged kaon restrict their absolute value. The strongest constraints come from $K_{L} \rightarrow \mu^{+} \mu^{-}\left[\operatorname{Re}\left(x_{\mu}\right)\right], K_{L} \rightarrow \pi^{0} \mu^{+} \mu-\left[\operatorname{Im}\left(x_{\mu}\right)\right], K_{L} \rightarrow \pi^{0} e^{+} e^{-}$ $\left[\operatorname{Im}\left(x_{e}\right)\right]$ and $K_{L} \rightarrow e^{+} e^{-}\left[\operatorname{Re}\left(x_{e}\right)\right]$.

More stringent constraints can be derived from the current experimental limits on LFV transitions. The 90\% CL upper bounds $\operatorname{Br}\left(K_{L} \rightarrow e^{ \pm} \mu^{\mp}\right)<4.7 \times 10^{-12}$ [2] and $\operatorname{Br}\left(K_{L} \rightarrow \pi^{0} e^{ \pm} \mu^{\mp}\right)<$ $7.6 \times 10^{-11}[61]$ imply [58]

$$
\begin{aligned}
& \left(\frac{1 \mathrm{TeV}}{M_{\mathrm{LQ}}}\right)^{2} \times\left\{\begin{array}{l}
\left|y_{\mathrm{LQ}}^{21}\left(y_{\mathrm{LQ}}^{12}\right)^{*}+y_{\mathrm{LQ}}^{22}\left(y_{\mathrm{LQ}}^{11}\right)^{*}\right| \\
\left|y_{\mathrm{LQ}}^{21}\left(y_{\mathrm{LQ}}^{12}\right)^{*}+y_{\mathrm{LQ}}^{11}\left(y_{\mathrm{LQ}}^{22}\right)^{*}\right|
\end{array} \mid 1.9 \times 10^{-5},\right. \\
& \left(\frac{1 \mathrm{TeV}}{M_{\mathrm{LQ}}}\right)^{2} \times\left\{\begin{array}{l}
\left|y_{\mathrm{LQ}}^{21}\left(y_{\mathrm{LQ}}^{12}\right)^{*}-y_{\mathrm{LQ}}^{22}\left(y_{\mathrm{LQ}}^{11}\right)^{*}\right| \\
\left|y_{\mathrm{LQ}}^{21}\left(y_{\mathrm{LQ}}^{12}\right)^{*}-y_{\mathrm{LQ}}^{11}\left(y_{\mathrm{LQ}}^{22}\right)^{*}\right|
\end{array} \mid 2.9 \times 10^{-4},\right.
\end{aligned}
$$


respectively, while $\operatorname{Br}\left(K^{+} \rightarrow \pi^{+} \mu^{+} e^{-}\right)<1.3 \times 10^{-11}$ [4] leads to

$$
\left(\frac{1 \mathrm{TeV}}{M_{\mathrm{LQ}}}\right)^{2} \times\left(\left|y_{\mathrm{LQ}}^{21}\left(y_{\mathrm{LQ}}^{12}\right)^{*}\right|,\left|y_{\mathrm{LQ}}^{11}\left(y_{\mathrm{LQ}}^{22}\right)^{*}\right|\right)<1.9 \times 10^{-4}
$$

for the four LQ types.

The $K \rightarrow \pi v \bar{v}$ decay modes only receive tree-level contributions from $S_{1}, S_{3}$ and $\tilde{R}_{2}$. For identical neutrino flavours $v_{\ell} \bar{v}_{\ell}$ in the final state, the corresponding constraints are shown in Fig. 9 [58]. The relevant combination of LQ parameters is in this case

$$
x_{v}=\left(\frac{1 \mathrm{TeV}}{M_{\mathrm{LQ}}}\right)^{2} \times \hat{y}_{\mathrm{LQ}}^{\ell \ell}\left(\hat{y}_{\mathrm{LQ}}^{2 \ell}\right)^{*}
$$

where $\hat{y}_{\mathrm{LQ}}=y_{\mathrm{LQ}} U$ with $U$ the PMNS neutrino mixing matrix. Notice that the neutral mode only constrains $\operatorname{Im}\left(x_{v}\right)$, while $K^{+} \rightarrow \pi^{+} v \bar{v}$ puts limits on both the real and imaginary parts of $x_{v}$.

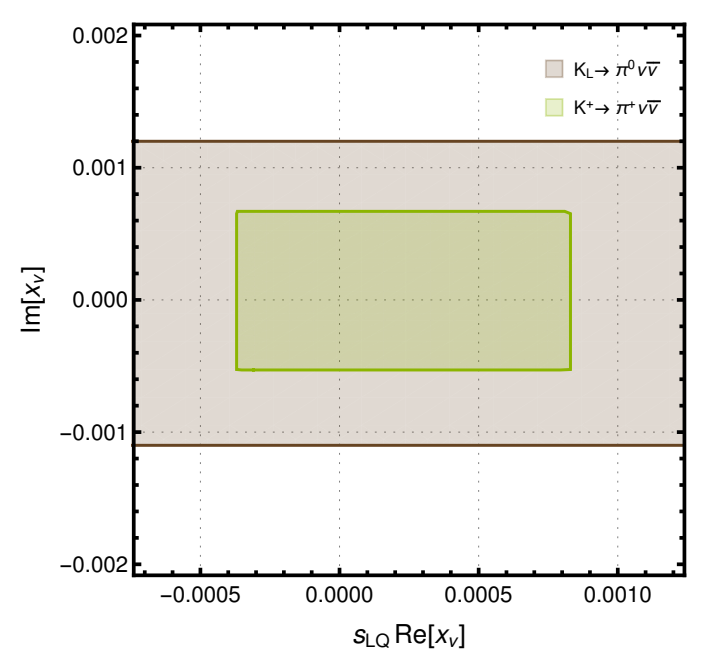

Figure 9: Allowed regions in the plane $\left(s_{\mathrm{LQ}} \operatorname{Re}\left[x_{v}\right], \operatorname{Im}\left[x_{v}\right]\right)$, arising from $K \rightarrow \pi v \bar{v}$ decays [58]. The sign factor $s_{\mathrm{LQ}}=+1$ for $S_{1,3}$, while $s_{\mathrm{LQ}}=-1$ for $\tilde{R}_{2}$.

The three LQs induce also $K \rightarrow \pi v_{m} \bar{v}_{n}$ decay modes with different neutrino flavours, which should not evade the experimental limits in Eq. (3.2). The $K^{+}$decay implies the upper bound [58]

$$
\left(\frac{1 \mathrm{TeV}}{M_{\mathrm{LQ}}}\right)^{2} \times\left[\sum_{m \neq n}\left|\hat{y}_{\mathrm{LQ}}^{1 m}\left(\hat{y}_{\mathrm{LQ}}^{2 n}\right)^{*}\right|^{2}\right]^{1 / 2}<6.0 \times 10^{-4},
$$

while the neutral decay mode puts the constraint [58]

$$
\left(\frac{1 \mathrm{TeV}}{M_{\mathrm{LQ}}}\right)^{2} \times\left[\sum_{m \neq n}\left|\hat{y}_{\mathrm{LQ}}^{1 m}\left(\hat{y}_{\mathrm{LQ}}^{2 n}\right)^{*}-\hat{y}_{\mathrm{LQ}}^{2 m}\left(\hat{y}_{\mathrm{LQ}}^{1 n}\right)^{*}\right|^{2}\right]^{1 / 2}<1.1 \times 10^{-3} .
$$

The KOTO collaboration has recently reported the observation of four $K_{L}^{0} \rightarrow \pi^{0} v \bar{v}$ events, with an expected background of only $0.05 \pm 0.02$ events [62]. Removing one of the events that is suspected to originate in underestimated upstream activity background, the quoted single event 
sensitivity of $6.9 \times 10^{-10}$ would correspond to $\operatorname{Br}\left(K_{L}^{0} \rightarrow \pi^{0} v \bar{v}\right) \sim 2 \times 10^{-9}$, well above the new Grossman-Nir limit [63] implied by the NA62 upper bound on $\operatorname{Br}\left(K^{+} \rightarrow \pi^{+} v \bar{v}\right)$ :

$$
\operatorname{Br}\left(K_{L}^{0} \rightarrow \pi^{0} v \bar{v}\right)<4.2 \times \operatorname{Br}\left(K^{+} \rightarrow \pi^{+} v \bar{v}\right)<7.8 \times 10^{-10} .
$$

This limit is valid under quite generic assumptions, provided the lepton flavour is conserved, and it can be directly inferred from the predicted LQ-induced decay amplitudes [58], if there are only identical neutrino flavours in the final state.

In order to reach the KOTO signal, one needs a sizeable decay amplitude into neutrinos with different flavours $(n \neq m)$. This could be easily achieved within the $S_{1}, S_{3}$ and $\tilde{R}_{2}$ LQ scenarios. A confirmation of the KOTO events would just imply that the combination of LQ couplings in Eq. (4.8) takes a non-zero value quite close to its current upper bound, indicating a violation of lepton flavour. Other possible NP interpretations have been already considered in Refs. [64, 65, 66, $67,68,69,70]$.

\section{Acknowledgements}

I want to thank the organizers of Beauty 2019 for the invitation to present this overview. I also thank V. Cirigliano, H. Gisbert, R. Mandal and A. Rodríguez-Sánchez for a very productive and enjoyable collaboration. This work has been supported in part by the Spanish Government and ERDF funds from the EU Commission [grant FPA2017-84445-P] and the Generalitat Valenciana [grant Prometeo/2017/053].

\section{References}

[1] V. Cirigliano, G. Ecker, H. Neufeld, A. Pich and J. Portoles, Kaon Decays in the Standard Model, Rev. Mod. Phys. 84 (2012) 399 [1107.6001].

[2] BNL collaboration, New limit on muon and electron lepton number violation from $K_{L}^{0} \rightarrow \mu^{ \pm} e^{\mp}$ decay, Phys. Rev. Lett. 81 (1998) 5734 [hep-ex/9811038].

[3] KTEV collaboration, Measurements of the Decay $K_{L} \rightarrow e^{+} e^{-} \mu^{+} \mu^{-}$, Phys. Rev. Lett. 90 (2003) 141801 [hep-ex/0212002].

[4] A. Sher et al., An Improved upper limit on the decay $K^{+} \rightarrow \pi^{+} \mu^{+} e^{-}$, Phys. Rev. D72 (2005) 012005 [hep-ex/0502020].

[5] R. Appel et al., Search for lepton flavor violation in $K^{+}$decays, Phys. Rev. Lett. 85 (2000) 2877 [hep-ex/0006003].

[6] F. J. Gilman and M. B. Wise, Effective Hamiltonian for $\Delta S=1$ Weak Nonleptonic Decays in the Six Quark Model, Phys. Rev. D20 (1979) 2392.

[7] A. J. Buras, M. Jamin, M. E. Lautenbacher and P. H. Weisz, Effective Hamiltonians for $\Delta S=1$ and $\Delta B=1$ nonleptonic decays beyond the leading logarithmic approximation, Nucl. Phys. $\mathbf{B 3 7 0}$ (1992) 69.

[8] A. J. Buras, M. Jamin, M. E. Lautenbacher and P. H. Weisz, Two loop anomalous dimension matrix for $\Delta S=1$ weak nonleptonic decays I: $\mathscr{O}\left(\alpha_{s}^{2}\right)$, Nucl. Phys. B400 (1993) 37 [hep-ph/9211304]. 
[9] A. J. Buras, M. Jamin and M. E. Lautenbacher, Two loop anomalous dimension matrix for $\Delta S=1$ weak nonleptonic decays. 2. $\mathscr{O}\left(\alpha \alpha_{s}\right)$, Nucl. Phys. B400 (1993) 75 [hep-ph/9211321].

[10] M. Ciuchini, E. Franco, G. Martinelli and L. Reina, The Delta $S=1$ effective Hamiltonian including next-to-leading order QCD and QED corrections, Nucl. Phys. B415 (1994) 403 [hep-ph/9304257].

[11] S. Weinberg, Phenomenological Lagrangians, Physica A96 (1979) 327.

[12] J. Gasser and H. Leutwyler, Chiral Perturbation Theory: Expansions in the Mass of the Strange Quark, Nucl. Phys. B250 (1985) 465.

[13] G. Ecker, Chiral perturbation theory, Prog. Part. Nucl. Phys. 35 (1995) 1 [hep-ph/9501357].

[14] A. Pich, Chiral perturbation theory, Rept. Prog. Phys. 58 (1995) 563 [hep-ph/9502366].

[15] NA31 collaboration, First Evidence for Direct CP Violation, Phys. Lett. B206 (1988) 169.

[16] Particle Data Group collaboration, Review of Particle Physics, Phys. Rev. D98 (2018) 030001.

[17] E. Pallante and A. Pich, Strong enhancement of $\varepsilon^{\prime} / \varepsilon$ through final state interactions, Phys. Rev. Lett. 84 (2000) 2568 [hep-ph/9911233].

[18] E. Pallante and A. Pich, Final state interactions in kaon decays, Nucl. Phys. B592 (2001) 294 [hep-ph/0007208].

[19] E. Pallante, A. Pich and I. Scimemi, The Standard model prediction for $\varepsilon^{\prime} / \varepsilon$, Nucl. Phys. B617 (2001) $441[$ hep-ph/0105011].

[20] H. Gisbert and A. Pich, Direct CP violation in $K^{0} \rightarrow \pi \pi$ : Standard Model Status, Rept. Prog. Phys. 81 (2018) 076201 [1712.06147].

[21] V. Cirigliano, H. Gisbert, A. Pich and A. Rodríguez-Sánchez, Isospin-Violating Contributions to $\varepsilon^{\prime} / \varepsilon$, 1911.01359.

[22] G. Ecker, G. Muller, H. Neufeld and A. Pich, $\pi^{0}-\eta$ mixing and CP violation, Phys. Lett. B477 (2000) 88 [hep-ph/9912264].

[23] V. Cirigliano, A. Pich, G. Ecker and H. Neufeld, Isospin violation in $\varepsilon^{\prime}$, Phys. Rev. Lett. 91 (2003) 162001 [hep-ph/0307030].

[24] V. Cirigliano, G. Ecker, H. Neufeld and A. Pich, Isospin breaking in $K \rightarrow \pi \pi$ decays, Eur. Phys. J. C33 (2004) 369 [hep-ph/ 0310351$].$

[25] RBC, UKQCD collaboration, Standard Model Prediction for Direct CP Violation in $K \rightarrow \pi \pi$ Decay, Phys. Rev. Lett. 115 (2015) 212001 [1505.07863].

[26] T. Blum et al., $K \rightarrow \pi \pi \Delta I=3 / 2$ decay amplitude in the continuum limit, Phys. Rev. D91 (2015) $074502[1502.00263]$.

[27] A. J. Buras and J.-M. Gérard, Upper bounds on $\varepsilon^{\prime} / \varepsilon$ parameters $B_{6}^{(1 / 2)}$ and $B_{8}^{(3 / 2)}$ from large $N Q C D$ and other news, JHEP 12 (2015) 008 [1507.06326].

[28] A. J. Buras and J.-M. Gerard, Final state interactions in $K \rightarrow \pi \pi$ decays: $\Delta I=1 / 2$ rule vs. $\varepsilon^{\prime} / \varepsilon$, Eur. Phys. J. C77 (2017) 10 [1603.05686].

[29] A. J. Buras, M. Gorbahn, S. Jäger and M. Jamin, Improved anatomy of $\varepsilon^{\prime} / \varepsilon$ in the Standard Model, JHEP 11 (2015) 202 [1507.06345]. 
[30] T. Kitahara, U. Nierste and P. Tremper, Singularity-free next-to-leading order $\Delta S=1$ renormalization group evolution and $\varepsilon_{K}^{\prime} / \varepsilon_{K}$ in the Standard Model and beyond, JHEP 12 (2016) 078 [1607.06727].

[31] G. D'Ambrosio and D. Espriu, Rare Decay Modes of the K Mesons in the Chiral Lagrangian, Phys. Lett. B175 (1986) 237.

[32] J. L. Goity, The Decays $K_{S}^{0} \rightarrow \gamma \gamma$ and $K_{L}^{0} \rightarrow \gamma \gamma$ in the Chiral Approach, Z. Phys. C34 (1987) 341.

[33] J. Kambor and B. R. Holstein, $K_{S} \rightarrow \gamma \gamma, K_{L} \rightarrow \pi^{0} \gamma \gamma$ and unitarity, Phys. Rev. D49 (1994) 2346 [hep-ph/9310324].

[34] G. Ecker and A. Pich, The Longitudinal muon polarization in $K_{L} \rightarrow \mu^{+} \mu^{-}$, Nucl. Phys. B366 (1991) 189.

[35] KLOE collaboration, Search for the $K_{S} \rightarrow e^{+} e^{-}$decay with the KLOE detector, Phys. Lett. $\mathbf{B 6 7 2}$ (2009) 203 [0811.1007].

[36] LHCB collaboration, Improved limit on the branching fraction of the rare decay $K_{\mathrm{S}}^{0} \rightarrow \mu^{+} \mu^{-}$, Eur. Phys. J. C77 (2017) 678 [1706.00758].

[37] LHCB collaboration, Strong constraints on the $K_{\mathrm{S}}^{0} \rightarrow \mu^{+} \mu^{-}$branching fraction, 2001.10354.

[38] G. Ecker, A. Pich and E. de Rafael, $K^{0} \rightarrow \pi^{0} \gamma \gamma$ Decays in Chiral Perturbation Theory, Phys. Lett. B189 (1987) 363.

[39] L. Cappiello and G. D'Ambrosio, $K_{L} \rightarrow \pi^{0} \gamma \gamma$ Decay in the Chiral Effective Lagrangian, Nuovo Cim. A99 (1988) 155.

[40] L. M. Sehgal, Rate and Spectrum of $K_{L} \rightarrow \pi^{0} \gamma \gamma$, Phys. Rev. D41 (1990) 161.

[41] NA48 collaboration, Precise measurement of the decay $K_{L} \rightarrow \pi^{0} \gamma \gamma$, Phys. Lett. B536 (2002) 229 [hep-ex/0205010].

[42] A. G. Cohen, G. Ecker and A. Pich, Unitarity and $K_{L} \rightarrow \pi^{0} \gamma \gamma$, Phys. Lett. B304 (1993) 347.

[43] L. Cappiello, G. D’Ambrosio and M. Miragliuolo, Corrections to $K \rightarrow \pi \gamma \gamma$ from $K \rightarrow 3 \pi$, Phys. Lett. B298 (1993) 423.

[44] G. Ecker, A. Pich and E. de Rafael, Vector Meson Exchange in Radiative Kaon Decays and Chiral Perturbation Theory, Phys. Lett. B237 (1990) 481.

[45] G. Ecker, A. Pich and E. de Rafael, Radiative Kaon Decays and CP Violation in Chiral Perturbation Theory, Nucl. Phys. B303 (1988) 665.

[46] G. D'Ambrosio and J. Portoles, Unitarity and vector meson contributions to $K^{+} \rightarrow \pi^{+} \gamma \gamma$, Phys. Lett. B386 (1996) 403 [hep-ph/ 9606213$].$

[47] NA48/2 collaboration, A new measurement of the $K^{ \pm} \rightarrow \pi^{ \pm} \gamma \gamma$ decay at the NA48/2 experiment, Phys. Lett. B730 (2014) 141 [1310 . 5499 ].

[48] J. F. Donoghue and F. Gabbiani, Reanalysis of the decay $K_{L} \rightarrow \pi^{0} e^{+} e^{-}$, Phys. Rev. D51 (1995) 2187 [hep-ph/9408390].

[49] A. J. Buras, M. E. Lautenbacher, M. Misiak and M. Munz, Direct CP violation in $K_{L} \rightarrow \pi^{0} e^{+} e^{-}$ beyond leading logarithms, Nucl. Phys. B423 (1994) 349 [hep-ph/ 9402347 ].

[50] G. Buchalla, G. D’Ambrosio and G. Isidori, Extracting short distance physics from $K_{L, S} \rightarrow \pi^{0} e^{+} e^{-}$ decays, Nucl. Phys. B672 (2003) 387 [hep-ph/ 0308008 ]. 
[51] KTEV collaboration, Search for the rare decay $K_{L} \rightarrow \pi^{0} e^{+} e^{-}$, Phys. Rev. Lett. 93 (2004) 021805 [hep-ex/0309072].

[52] A. J. Buras, M. Gorbahn, U. Haisch and U. Nierste, The Rare decay $K^{+} \rightarrow \pi^{+} v \bar{v}$ at the next-to-next-to-leading order in QCD, Phys. Rev. Lett. 95 (2005) 261805 [hep-ph/ 0508165$].$

[53] J. Brod, M. Gorbahn and E. Stamou, Two-Loop Electroweak Corrections for the $K \rightarrow \pi \nu \bar{v}$ Decays, Phys. Rev. D83 (2011) 034030 [1009.0947].

[54] M. Gorbahn, "Latest results of the $K \rightarrow \pi \nu \bar{v}$ branching ratio calculations." Talk at Kaon 2019, Perugia (Italy), September 10th, 2019.

[55] NA62 collaboration, G. Ruggiero, "New result on $K^{+} \rightarrow \pi^{+} v \bar{v}$ from the NA62 experiment." Talk at Kaon 2019, Perugia (Italy), September 10th, 2019.

[56] KOTO collaboration, Search for the $K_{L} \rightarrow \pi^{0} v \bar{v}$ and $K_{L} \rightarrow \pi^{0} X^{0}$ decays at the J-PARC KOTO experiment, Phys. Rev. Lett. 122 (2019) 021802 [1810.09655].

[57] A. Pich, Flavour Anomalies, PoS LHCP2019 (2019) 078 [1911. 06211].

[58] R. Mandal and A. Pich, Constraints on scalar leptoquarks from lepton and kaon physics, JHEP 12 (2019) 089 [1908.11155].

[59] S. Davidson, D. C. Bailey and B. A. Campbell, Model independent constraints on leptoquarks from rare processes, Z. Phys. C61 (1994) 613 [hep-ph/9309310].

[60] I. Doršner, S. Fajfer, A. Greljo, J. F. Kamenik and N. Košnik, Physics of leptoquarks in precision experiments and at particle colliders, Phys. Rept. 641 (2016) 1 [1603.04993].

[61] KTEV collaboration, Search for lepton flavor violating decays of the neutral kaon, Phys. Rev. Lett. 100 (2008) 131803 [0711.3472].

[62] KOTO collaboration, S. Shinohara, "Search for the rare decay $K_{L} \rightarrow \pi^{0} v \bar{v}$ at J-PARC KOTO experiment.” Talk at Kaon 2019, Perugia (Italy), September 10th, 2019.

[63] Y. Grossman and Y. Nir, $K_{L} \rightarrow \pi^{0} v \bar{v}$ beyond the standard model, Phys. Lett. B398 (1997) 163 [hep-ph/9701313].

[64] T. Kitahara, T. Okui, G. Perez, Y. Soreq and K. Tobioka, New physics implications of recent search for $K_{L} \rightarrow \pi^{0} v \bar{v}$ at KOTO, 1909.11111 .

[65] D. Egana-Ugrinovic, S. Homiller and P. Meade, Light Scalars and the KOTO Anomaly, 1911.10203.

[66] P. S. B. Dev, R. N. Mohapatra and Y. Zhang, Constraints on long-lived light scalars with flavor-changing couplings and the KOTO anomaly, 1911.12334.

[67] M. Fabbrichesi and E. Gabrielli, Dark-sector physics in the search for the rare decays $K^{+} \rightarrow \pi^{+} \bar{v} v$ and $K_{L} \rightarrow \pi^{0} \bar{v} v, 1911.03755$.

[68] T. Li, X.-D. Ma and M. A. Schmidt, Implication of $K \rightarrow \pi v \bar{v}$ for generic neutrino interactions in effective field theories, 1912.10433.

[69] Y. Jho, S. M. Lee, S. C. Park, Y. Park and P.-Y. Tseng, Light gauge boson interpretation for $(g-2)_{\mu}$ and the $K_{L} \rightarrow \pi^{0}+($ invisible) anomaly at the J-PARC KOTO experiment, 2001.06572.

[70] J. Liu, N. McGinnis, C. E. M. Wagner and X.-P. Wang, A Light Scalar Explanation of $(g-2)_{\mu}$ and the KOTO Anomaly, 2001.06522. 